\title{
Optimization Algorithm of Infrared-Polarization Image Fusion Based on Fireworks Algorithm
}

\author{
Zhiyong Yang \\ Xi'an Research Institute of High-tech \\ Gaoxiang Lu ( lu894118008@163.com ) \\ Xi'an Research Institute of High-tech \\ Wei Cai \\ Xi'an Research Institute of High-tech \\ Danqiu Qiao \\ Xi'an Research Institute of High-tech \\ Junchen Song \\ Xi'an Research Institute of High-tech
}

\section{Research Article}

Keywords: Firework algorithm, Intelligent optimization algorithm, Polarization imaging, Image fusion

Posted Date: February 17th, 2021

DOI: https://doi.org/10.21203/rs.3.rs-199225/v1

License: (c) (i) This work is licensed under a Creative Commons Attribution 4.0 International License. Read Full License 


\title{
Optimization Algorithm of Infrared-Polarization Image Fusion Based on Fireworks Algorithm
}

\author{
Zhiyong Yang ${ }^{1}$, Gaoxiang Lu ${ }^{1 \varpi}$, Wei Cai ${ }^{1}$, Danqiu Qiao', Junchen Song ${ }^{1}$
}

\begin{abstract}
\end{abstract}
Because of the shortcomings of traditional infrared-polarization image fusion algorithm, such as low intelligence and single optimization index, this paper proposes an intelligent infrared-polarization image fusion optimization algorithm based on fireworks algorithm. Firstly, an improved differential image correction method based on single pixel nonuniformity is proposed to remove the cold reflection. The two-dimensional discrete cosine transform (DCT) is used to reduce the image sensitivity and improve the robustness, and the Stokes vector formula is used to obtain the polarization characteristic image. Secondly, based on the strong complementarity between infrared-intensity image and degree of linear-polarization (DOLP) image and the explosive optimization of fireworks algorithm, the problem model of weighted fusion algorithm is established, and the fitness function based on root mean square error (RMSE) is constructed to calculate the optimal weight of source image. In the fusion experiment of long-wave infrared-intensity image and DOLP image, this method is compared with the common fusion algorithms. The results show that this method can effectively fuse the infrared-intensity and degree of polarization information, and the evaluation indexes of standard deviation, spatial frequency, mutual information, structural similarity, peak signal-to-noise ratio and information entropy of the fusion image are better than the comparison 1Xi'an Research Institute of High-tech, Xi'an, Shannxi 710025, China

๒email: lu894118008@163.com. 
algorithm. In the future, cooperated with the long-wave infrared-polarization imaging system, this method can be applied to improve the infrared detection ability in complex environment.

Keywords: Firework algorithm, Intelligent optimization algorithm, Polarization imaging, Image fusion

\section{Introduction}

For a long time, infrared imaging technology is a powerful means of tactical reconnaissance in modern war, but with the increasingly complex battlefield environment, the traditional infrared imaging technology is difficult to meet the needs of new photoelectric countermeasures in complex environment. Infrared-polarization imaging detection can obtain polarization information different from the background according to the target characteristics, and make up for the shortcomings of traditional imaging technology by adding polarization dimension information ${ }^{1}$. Therefore, the fusion of infrared-intensity image and DOLP image can improve the ability of target detection and recognition, which plays an important role in weapon reconnaissance and anti-reconnaissance. How to describe the target characteristics accurately through the rich scene information of fusion images is the key problem in the process of image fusion.

Scholars at home and abroad have carried out a lot of research on infrared polarization image fusion. For instance, Zhang $^{2}$ used unsupervised depth network to solve the problem of infrared-polarization image fusion; Zhang $^{3}$ proposed a method based on multi-scale Gaussian filter and residual to fuse infrared polarization and intensity images. The fusion results retain all the features of infrared-intensity image and most of the features of DOLP image. In the case of 
low contrast between target and background, the performance of common fusion algorithm in target recognition is not improved much. $\mathrm{Kong}^{4}$ proposed an infrared-polarization image fusion algorithm based on cross structure regularization to enhance the visual effect and visual quality of infrared-polarization image.

In general image fusion algorithms, the weight of source image is determined by setting a threshold value, and the consistency of evaluation indexes is poor. To solve this problem, some scholars proposed an intelligent fusion algorithm based on maximizing the evaluation index to obtain the weight of the source image. $\mathrm{Kanwal}^{5}$ proposed an intelligent multi-scale exposure fusion algorithm based on image segmentation to preserve the details of the darkest and brightest areas. Dai ${ }^{6}$ proposed a fusion method of infrared image and visible image, which can realize the optimization of fuzzy region feature frame. The performance of different intelligent fusion algorithms is very different. For example, when the acceleration constant of particle swarm optimization algorithm ${ }^{7}$ is different, it may only be local optimal, and flower pollination algorithm $^{8}$ is easy to fall into "dimension disaster" when solving high-dimensional optimization problems. In order to have the same advantages in terms of convergence speed and computing performance, Tan Ying from Peking University proposed the Fireworks Algorithm ${ }^{9}$ (FWA).

Since the fireworks algorithm was proposed, it has successfully solved the optimization problems in different fields, such as image recognition ${ }^{10}$, image segmentation ${ }^{11}$, etc. Zheng ${ }^{12}$ proposed the enhanced fireworks algorithm, which effectively enriched the diversity of the population; $\mathrm{Lu}^{13}$ proposed a fireworks algorithm based on dual population strategy, which improved the efficiency and accuracy of system fault diagnosis. Cui ${ }^{14}$ proposed a hyperspectral 
image classification method based on dimension reduction of fireworks algorithm, which reduced the amount of hyperspectral data and computational complexity, and avoided Hughes phenomenon in subsequent classification; Beijinariu ${ }^{15}$ successfully applied fireworks algorithm to multispectral image fusion.

In view of the excellent performance of fireworks algorithm in the field of image processing, this paper introduces fireworks algorithm into infrared-polarization image fusion process, and proposes an intelligent fusion algorithm of infrared-polarization image based on fireworks algorithm. Firstly, the polarization source image is preprocessed to improve the robustness of the image, and then the optimization problem model is proposed based on the weighted fusion idea of the source image. According to the root mean square error (RMSE) of the infrared-intensity image, the DOLP image and the fused image, the fitness function is constructed to calculate the optimal weight of the source image. The experimental results show that the algorithm introduced in this paper has a higher degree of intelligence, by which several image fusion indexes have been achieved at their best, and the image fusion quality and visual effect get greatly improved.

\section{Image fusion ideas and preprocessing}

\subsection{Fusion ideas}

The general idea of the fusion algorithm in this paper is as follows: obtain the polarization image $I_{\theta}\left(\theta=0^{\circ}, 45^{\circ}, 90^{\circ}, 135^{\circ}\right)$, remove the cold reflection through single-point non-uniformity correction difference, then sharpen the image through the Discrete cosine transform, get four Stokes parameter images after calculating, and calculate the DOLP image 
according to the Stokes vector formula; obtain the fusion weight of infrared-intensity image and polarization degree image is based on the optimization principle of root mean square error, then fuse the source image, and evaluate the fused image objectively, as well as subjectively. Figure 1 is the flowchart of infrared-polarization image fusion based on firework algorithm

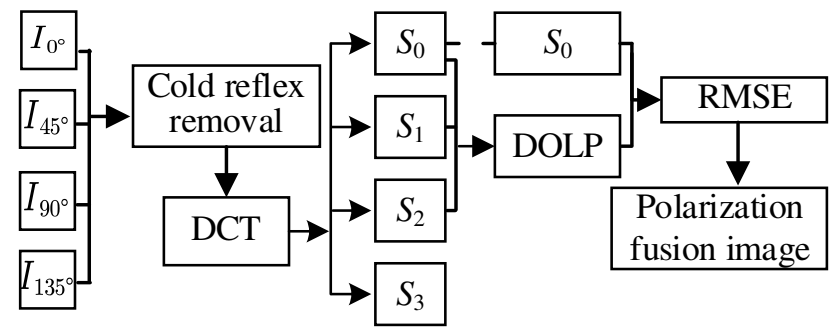

Figure 1. Flow chart of infrared-polarization image fusion based on firework algorithm

Where, $S_{0}$ represents light intensity information; $S_{1}$ is the intensity difference of linear polarization in $0^{\circ}$ and $90^{\circ}$ directions; $S_{2}$ is the intensity difference of linear polarization in $45^{\circ}$ and $135^{\circ}$ directions; $S_{3}$ is circular polarization information which is a small value in natural light, where it is assumed to be 0 .

\subsection{Image preprocessing}

\subsubsection{Removal of cold reflection}

There are significant differences and complementarities between the infrared-intensity image and the DOLP image. However, because the long-wave infrared images used in the experimental system are uncooled thermal images, so when the polarizer is placed parallel to the optical lens of the uncooled infrared thermal imager, there is a large temperature difference among the thermal imager, the infrared polarizer and the lens tube, thus forming a cold reflection, the direct fusion of the two images will affect the analysis of the polarization characteristics of the target. 
The cold reflection becomes a white bright spot in the infrared-polarization image, which seriously affects the image quality, as shown in Figure 2.

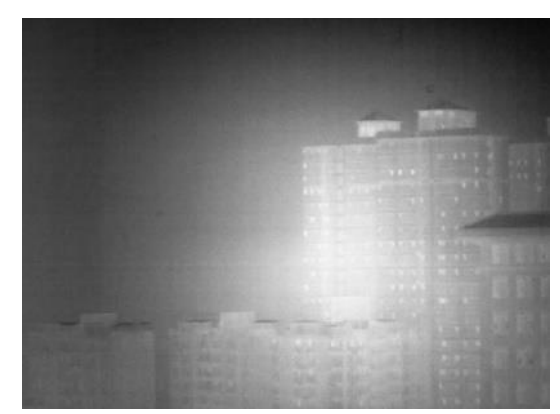

Figure 2 Cold reflection phenomenon of long-wave infrared-polarization imaging

In order to remove the irremovable vignetting effect signal superimposed on the infrared-polarization image, this paper proposes an improved correction method based on the single-pixel non-uniformity differential image. When the initial position is set as $0^{\circ}$ and the polarizer is rotated to the $\theta$ position, the target scene image with cold reflection and the cold reflection reference image are collected. $I_{\text {base }}$ is the cold reflection reference image, and $I_{\text {offset }}$ is the cold reflection correction image. The cold reflection reference image decomposed into high and low frequency, and the single point nonuniformity correction carried out respectively.

The single-point nonuniformity correction formula is as follows:

$$
I_{\text {offet }}^{\theta}(i, j)=I_{\text {base }}^{\theta}(i, j)-\sum_{i=1}^{n} \sum_{i=1}^{m} I_{\text {base }}^{\theta}(i, j) /(m \cdot n)
$$

Where, $n$ and $m$ are the number of pixels in the detector row and column, and $(i, j)$ is the coordinates of each position in the imaging image.

The target scene images with cold reflection are decomposed by high and low frequency, and then the cold reflection correction images are respectively differentiated, and the accurate linearly polarized images are obtained by reconstruction. $I_{\text {objective }}$ is the target scene image 
with cold reflection, and $I_{\text {amend }}$ is the target removing cold reflection image. The formula is as follows:

$$
I_{\text {amend }}^{\theta}(i, j)=I_{\text {objective }}^{\theta}(i, j)-I_{\text {offet }}^{\theta}(i, j)
$$

\subsubsection{Image sharpening}

The experimental data are obtained by the long-wave infrared time-sharing polarization imaging system. The quality of the single frame image obtained by this system is much higher than that obtained by the polarization focal plane imaging system and the polarization aperture polarization imaging system. However, due to the vibration of the rotating table, the polarization information calculation will be interfered. For the sensitivity of infrared-polarization imaging system, it is necessary to sharpen the source image. Sharpening method: decompose the polarization image with cold reflection removed by DCT, combine the high and low frequency coefficient images according to the visual saliency, and finally carry out the image inverse transformation. The formulas of DCT forward transform and inverse transform are as follows:

$$
\begin{gathered}
F(u, v)=\alpha(u, v) \sum_{x=0}^{M-1} \sum_{y=0}^{N-1} f(x, y) \cos \frac{(2 x+1) u \pi}{2 M} \cdot \cos \frac{(2 y+1) v \pi}{2 N} \\
f(x, y)=\sum_{x=0}^{M-1} \sum_{y=0}^{N-1} \alpha(u, v) F(u, v) \cos \frac{(2 x+1) u \pi}{2 M} \cdot \cos \frac{(2 y+1) v \pi}{2 N} \\
\text { Where, } \alpha(u)=\left\{\begin{array}{l}
\frac{1}{\sqrt{M}}, u=0 \\
\sqrt{\frac{2}{M}}, u \neq 0
\end{array}, \alpha(v)=\left\{\begin{array}{l}
\frac{1}{\sqrt{N}}, v=0 \\
\sqrt{\frac{2}{N}}, v \neq 0
\end{array}\right.\right.
\end{gathered}
$$

Positive transformation: $x=0,1,2 \ldots M-1 ; y=0,1,2 \ldots N-1$ 
Inverse transformation: $u=0,1,2 \ldots M-1 ; v=0,1,2 \ldots N-1$

\subsubsection{Calculation of polarization information}

The polarization state of long-wave infrared can be expressed by Stokes parameter, and its expression formula is as follows:

$$
S=\left[\begin{array}{c}
S_{0} \\
S_{1} \\
S_{2} \\
S_{3}
\end{array}\right]=\left[\begin{array}{l}
\left|E_{x}\right|^{2}+\left|E_{y}\right|^{2} \\
\left|E_{x}\right|^{2}-\left|E_{y}\right|^{2} \\
2\left|E_{x}\right|\left|E_{y}\right| \cos \delta \\
2\left|E_{x}\right|\left|E_{y}\right| \sin \delta
\end{array}\right]
$$

The DOLP image is a scalar value between 0 and 1 , which is defined as follows:

$$
D O L P=\frac{\sqrt{S_{1}^{2}+S_{2}^{2}}}{S_{0}}
$$

In the experiment, the infrared-polarization images are usually obtained in the polarization directions of $0^{\circ}, 45^{\circ}, 90^{\circ}$ and $135^{\circ}$ respectively. Therefore, the Stokes vector can be obtained directly from the following formula:

$$
S=\left[\begin{array}{c}
S_{0} \\
S_{1} \\
S_{2} \\
S_{3}
\end{array}\right]=\left[\begin{array}{c}
I_{0^{\circ}}+I_{90^{\circ}} \\
I_{0^{\circ}}-I_{90^{\circ}} \\
I_{45^{\circ}}-I_{135^{\circ}} \\
I_{L C}-I_{R C}
\end{array}\right]
$$

Where, $I_{L C}$ is the left-handed polarized light, and $I_{L C}$ is the right-handed polarized light.

\section{Image fusion}

Fireworks algorithm, as a new swarm intelligence optimization algorithm, uses explosion search mechanism to simulate the possible solution of fireworks optimization problem, takes the location of fireworks explosion as the center to generate a certain number of sparks within the set range, and selects the sparks within the optimal range to ignite the next generation of 
fireworks through the defined fitness function. Sparks fitting the fitness function are searched in the center of fireworks, and the next generation fireworks are detonated to generate multiple iterative optimization effects. In this process, information sources and interactive information are distributed intelligently, and the optimal solution of the solution space is finally obtained. In this paper, the fireworks algorithm is applied to the fusion of infrared-intensity image and DOLP image. The main steps of the algorithm include problem model, fitness function and algorithm flow.

(1) Problem model

Infrared-polarization image fusion usually obtains the weights of infrared-intensity image and DOLP image according to the fusion rules, and then fuses the source image through the weight matrix.

$$
I_{f}=\eta_{1} I_{l}+\eta_{2} I_{p}
$$

Where, $I_{f}$ is the fused image, $I_{l}$ is the infrared-intensity image, $I_{p}$ is the DOLP image, $\eta_{1}$ and $\eta_{2}$ is the weight of the source image. In order to prevent the energy spillover of the fusion image, the weighted average method is introduced, so Equation (8) is rewritten as:

$$
I_{f}=\left(\eta_{1} I_{l}+\eta_{2} I_{p}\right) /\left(\eta_{1}+\eta_{2}\right)
$$

The weighted average method affects the quality of the fused image by controlling the weight of the source image. Therefore, the essence of image fusion is the optimization of the weight of the source image. In the fireworks algorithm, the optimal solution is the parameter value of the fitness function minimization. The optimization problem model is represented by the following formula: 


$$
\min f\left(x_{1}, x_{2}, \ldots, x_{n}\right), 0<x_{i}<1
$$

Where, $f\left(x_{1}, x_{2}, \ldots, x_{n}\right)$ is the fusion image quality evaluation relation, and is also the fitness function of the optimization problem of the weighted function. $0<x_{i}<1$ represents the constraint condition, and $\left(x_{1}, x_{2}, \ldots x_{n}\right)$ is the $n$-dimensional optimization variable.

(2) Fitness function

The RMSE is an index to measure the deviation of experimental data from the real value, which is used to characterize the difference of gray information distribution between the source image and the fused image. RMSE is expressed by the following formula:

$$
R M S E=\sqrt{\frac{1}{M \cdot N} \sum_{i=1}^{M} \sum_{j=1}^{N}(X(i, j)-Y(i, j))^{2}}
$$

Where, $X(i, j)$ is the gray value of current image point $(i, j), Y(i, j)$ is the gray value of reference image point $(i, j)$, and $M$ and $N$ are the height and width of the image respectively. In the process of image fusion, the more information the fusion image extracts from the source image, the better the fusion effect. The smaller the RMSE value is, the smaller the difference between the two images is, indicating that the fused image retains more information from the source image. Based on the above analysis, the fitness function of the optimization problem is constructed by using the root mean square error of the gray values of the two images.

According to the definition of fitness function, the smaller the fitness value is, the closer it is to the solution of the optimization problem. The fusion of infrared-polarization image is composed of one infrared-intensity image and one DOLP image. Due to the different contribution of the source image to the fusion image evaluation index, a comprehensive root 
mean square error function is established for the two source images and the fusion image, and the comprehensive root mean square error function is expressed as:

$$
R M S E_{F L P}=\left(v_{1} R M S E_{F L}+v_{2} R M S E_{F P}\right) / 2
$$

Where, $R M S E_{F L}$ is the root mean square error of the fused image $F$ and the infrared-intensity image $L ; R M S E_{F P}$ is the root mean square error of the fused image $F$ and the DOLP image $P ; v_{1}$ and $v_{2}$ respectively represent the contribution of the infrared-intensity image and the DOLP image to the comprehensive root mean square error $R M S E_{F L P}$. According to the problem model and the comprehensive root mean square error function), $R M S E_{F L P}$ can be expressed as:

$$
R M S E_{F L P}=f\left(\eta_{1}, \eta_{2}, v_{1}, v_{2}\right)
$$

Therefore, equation (14) is selected as the fitness function of image fusion optimization problem. The weights $\eta_{1}$ and $\eta_{2}$ are introduced into the problem model, and the fitness function is constructed to introduce the contribution weights $v_{1}$ and $v_{2}$. Therefore, the problem space dimension suitable for fireworks algorithm is 4 . The optimal weights $\eta_{1}$ and $\eta_{2}$ needed for fusion image are obtained by calculation. Finally, the fusion image of the infrared-intensity image and the DOLP image with the best fitness value is obtained by fusion.

(3) Process of optimization algorithm

Let $W=\left\{\eta_{i}, i=1,2, \cdots, N\right\}$ be the population of $N$ fireworks, and $\eta_{i}=\left(\eta_{i}^{1}, \eta_{i}^{2}, \eta_{i}^{3}, \eta_{i}^{4}\right)$ is the position of individual fireworks $\eta_{i}$ in the 4-dimensional problem domain space. The algorithm process is simplified as follows:

1) Initialize the system and set parameters, select $N$ locations randomly, and assume the 
initial number of fireworks in this paper is 10 .

2) Ten fireworks were detonated at different positions. The sparks produced by the fireworks explosion are shown in Figure 3.

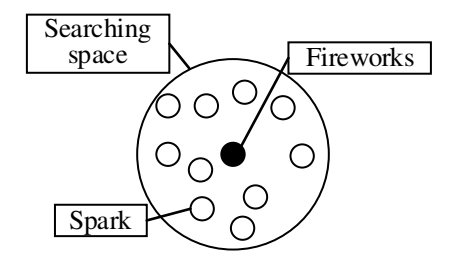

Figure 3. Sparks from fireworks explosions

3) Calculate the fitness values of all fireworks explosions according to the fitness function.

4) Calculate the number of sparks generated by the explosion of each firework $\eta_{i}$, which is defined as follows:

$$
S_{i}=m \cdot \frac{f_{\max }-f\left(\eta_{i}\right)+\sigma}{\sum_{i=1}^{n}\left(f_{\max }-f\left(\eta_{i}\right)\right)+\sigma}
$$

Where, $m$ is the parameter controlling the number of sparks generated by fireworks explosion in each generation, $f_{\max }=\max f\left(\eta_{i}\right)(i=1,2, \ldots, n)$ is the fitness value of the individual with the worst performance in the current population, and $\sigma$ is a minimum constant to prevent errors in the operation of the formula.

5) Calculate the explosion radius $A_{i}$ generated by the $i$-th fireworks explosion, which is defined as follows:

$$
A_{i}=A \cdot \frac{f\left(\eta_{i}\right)-f_{\min }+\sigma}{\sum_{i=1}^{n}\left(f\left(\eta_{i}\right)-f_{\min }\right)+\sigma}
$$

Where, $\mathrm{A}$ is the maximum explosion radius, $f_{\min }=\min f\left(\eta_{i}\right)$ is the fitness value of the individual with the optimal performance in the current population. 
6) Fireworks explode to produce $\eta_{i}$ regular sparks, and calculate the fitness of all sparks in the current group.

7) In order to improve the diversity of the population, Gaussian variation $\operatorname{spark}^{16}$ is introduced. The formula is:

$$
P_{y h}=P_{y h}+\left(P_{b h}-P_{y h}\right) e
$$

Where, $e$ is a random variable of Gaussian distribution, $P_{b h}$ is the position information of the fireworks with the smallest fitness value in the current population in the H-dimensional entropy, and $P_{y h}$ is the position information of the $y$-th fireworks in the H-dimensional entropy.

8) Calculate the fitness values of all types of fireworks, and divide them into three kinds of fireworks according to the fitness values of fireworks: good, medium and bad, and take 1/3 of each part to form a new generation of sparks. The position where the individual reaches the optimal fitness value is the solution of the optimization problem. Set the optimal fitness value, and stop running when the algorithm reaches the optimal fitness value, otherwise go to Step 2 and continue to execute until the last iteration. The flow chart of the optimization algorithm is shown in figure 4. 


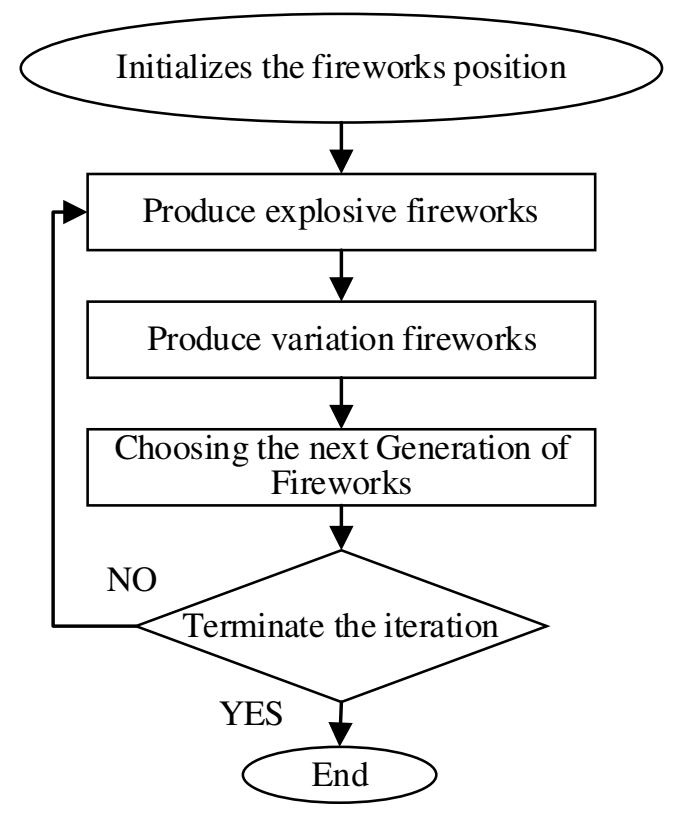

Figure 4. Flow chart of optimization algorithm

Algorithm iteration is an important feature of fireworks algorithm, which can decide whether to continue the iteration step according to the maximum number of iterations or the best fitness value. In order to optimize the algorithm flow and reduce the amount of calculation, the principle of maximum iteration number is adopted. In this algorithm, the variable dimension is set to 4 dimensions, and the maximum number of iterations is 100 . All new sparks are evaluated by fitness function, and the best, the worst and the other two randomly selected sparks are selected to form the next generation fireworks. As can be seen from figure 5, the fireworks algorithm can converge quickly and accurately when searching for the group optimal solution. 


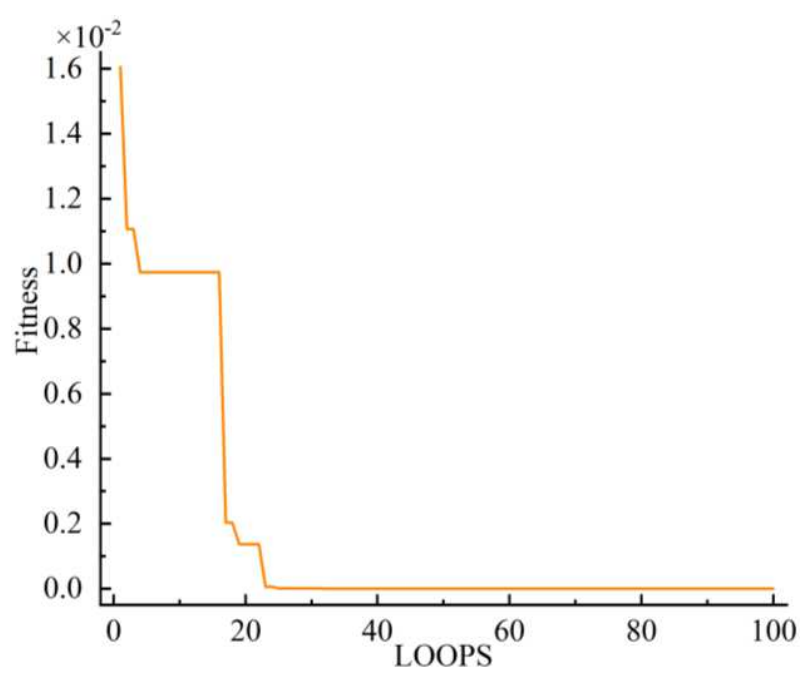

Figure 5. Optimization process of RMSE fitness

\section{Experiment and quality analysis}

Fireworks algorithm is to simulate the process of spark ignition fireworks to get the optimal solution. In order to verify the effectiveness of the fitness function based on root mean square error in solving the fusion weight problem and evaluate the image fusion ability of fireworks algorithm, this paper obtains the infrared-intensity image and the DOLP image through the laboratory equipment long-wave infrared-polarization imaging system, after image preprocessing work such as removing the cold reflection and reducing the image sensitivity, and fuses the DOLP image. At the same time, in order to evaluate the performance of the proposed algorithm, the proposed method is compared with other fusion methods.

\subsection{Experimental environment}

Experimental environment: Intel Core i7-5500u, 3.0GHz, 12GB memory, 64-bit win 10 operating system and MATLAB 2019a running software. Main parameters of polarization imaging system: The detection wavelength ranges from $7500 \mathrm{~nm}$ to $14000 \mathrm{~nm}$, and the extinction ratio of polarizer is 10000:1. 


\subsection{Image objective evaluation index}

The objective evaluation of image is mainly composed of the following 6 indicators to evaluate the results of image processing and fusion quantitatively.

(1) Average gradient (AG) is an evaluation index describing the gradient information of the fused image. The larger the Average gradient value is, the clearer the image details and texture information will be. The calculation formula is as follows:

$$
A G=\frac{1}{M \cdot N} \sum_{i=1}^{M} \sum_{j=1}^{N} \sqrt{\frac{(F(i, j)-F(i+1, j))^{2}+(F(i, j)-F(i, j+1))^{2}}{2}}
$$

Where, $F(i, j)$ is the gray value of the pixel points in the $i$ row and $j$ column of the image.

(2) Standard deviation (STD) is an evaluation index to describe the gray level distribution and contrast of fused image. The larger the Standard deviation is, the more kinds of fused image information are. The formula is as follows:

$$
S T D=\sqrt{\frac{\sum_{i=1}^{M} \sum_{j=1}^{N}(F(i, j)-\mu)^{2}}{M \cdot N}}
$$

Where, $M$ and $N$ respectively represent the height and width of the image, $\mu$ is the gray mean of the fusion image.

(3) Spatial frequency (SF) reflects the richness of the image spectrum. The formula is as follows:

$$
S F=\sqrt{R F^{2}+C F^{2}}
$$

Where, $R F$ and $C F$ correspond to image row and column frequencies respectively.

(4) Information entropy (IE) is used to calculate the average Information quantity of the fused image. The bigger the Information Entropy is, the bigger the average Information 
quantity is. The formula is as follows:

$$
I E=-\sum_{i=0}^{255} p(i) \log _{2} p(i)
$$

Where, $p(i)$ is the proportion of pixels with gray value of in the image.

(5) Peak signal to noise ratio (PSNR) is a full reference image quality evaluation index.

The calculation formula is as follows:

$$
P S N R=20 \log _{10}\left(\frac{2 n-1}{R M S E}\right)
$$

Where, $n$ is the number of bits per pixel, which is 8 in this paper. The larger the Peak signal to noise ratio, the smaller the distortion is, which can be used to evaluate the quality of image fusion.

(6) Mutual information (MI) reflects the degree of association between the source image and the fused image, which is expressed by the following formula:

$$
M I=\sum_{i=1}^{L} \sum_{j=1}^{L} h_{X, F}(i, j) \cdot \log _{2} \frac{h_{X, F}(i, j)}{h_{X}(i) \cdot h_{F}(j)}
$$

Where, $h_{X, F}(i, j)$ is the normalized joint histogram distribution formed by the source image $X$ and the fusion image $F ; h_{X}(i)$ and $h_{F}(j)$ represent normalized edge histogram distribution of source image and fusion image respectively; $L$ is the gray scale series.

(7) Structural similarity index measure (SSIM) is an index to evaluate the loss and distortion degree of the fused image, and its calculation formula is as follows:

$$
S S I M_{X, F}=\sum_{x, f} \frac{2 \mu_{x} \mu_{f}+C_{1}}{\mu_{x}^{2}+\mu_{f}^{2}+C_{1}} \cdot \frac{2 \sigma_{x f}+C_{2}}{\sigma_{x}^{2}+\sigma_{f}^{2}+C_{2}}
$$

Where, $\mu_{x}$ and $\mu_{f}$ respectively represent the gray values of image $X$ and fused image $F$; 
$\sigma_{x}$ and $\sigma_{f}$ respectively represent the standard deviation of image $X$ and fused image $F ; \sigma_{x f}$ is the gray covariance of source image $X$ and fused image $F$. The function of parameters $C_{1}$ and $C_{2}$ is to keep the algorithm stable, and their values are usually set as 0 . The greater the structural similarity is, the smaller the SSIM between the two images is, and the more information inherited from the source image. In this paper, the structural similarity index is the sum of the structural similarity values of the fused image and the two source images.

\subsection{Results of image preprocessing}

Figure.6(a) is the original infrared-polarization image, and Figure.6(b) is the infrared-polarization image corrected by removing the cold reflection. There is obvious cold reflection in the infrared-polarization image, and an obvious white spot in the center of the field of vision. From the experimental results, the cold reflection spot of the infrared target scene is almost eliminated. In the 3-dimensional gray image, the target shows the original infrared characteristics after removing the cold reflection, which shows that the purpose of preprocessing can be achieved by removing the cold reflection effect.

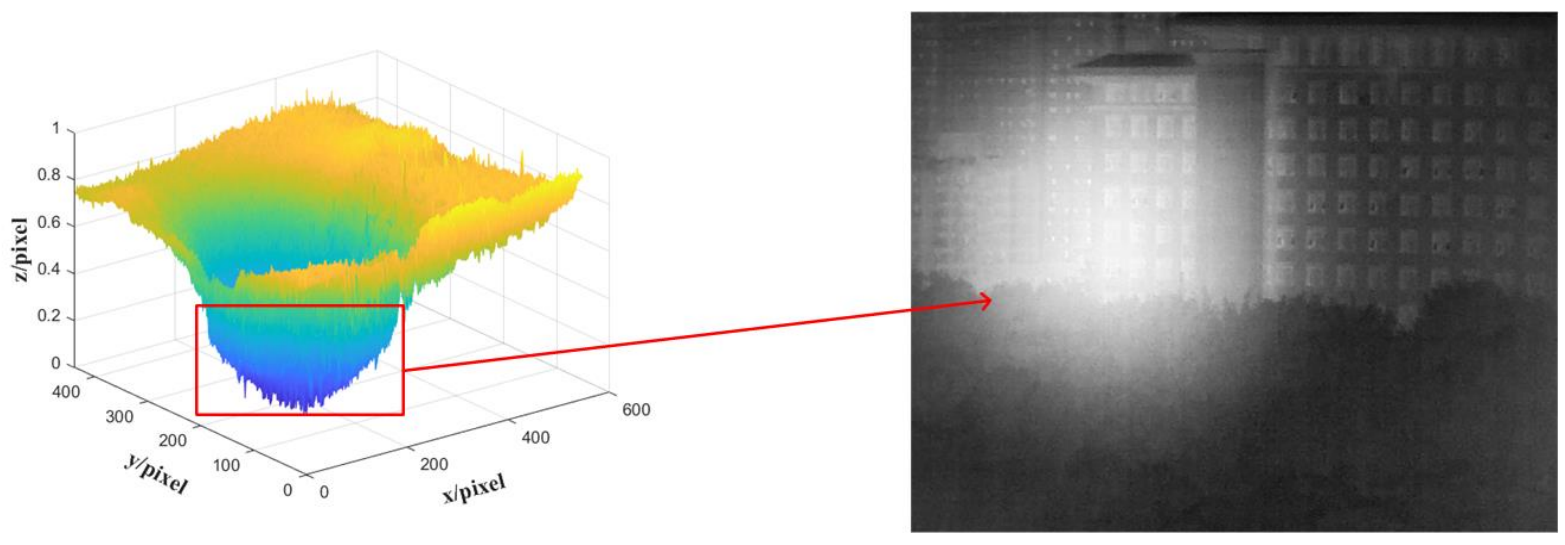




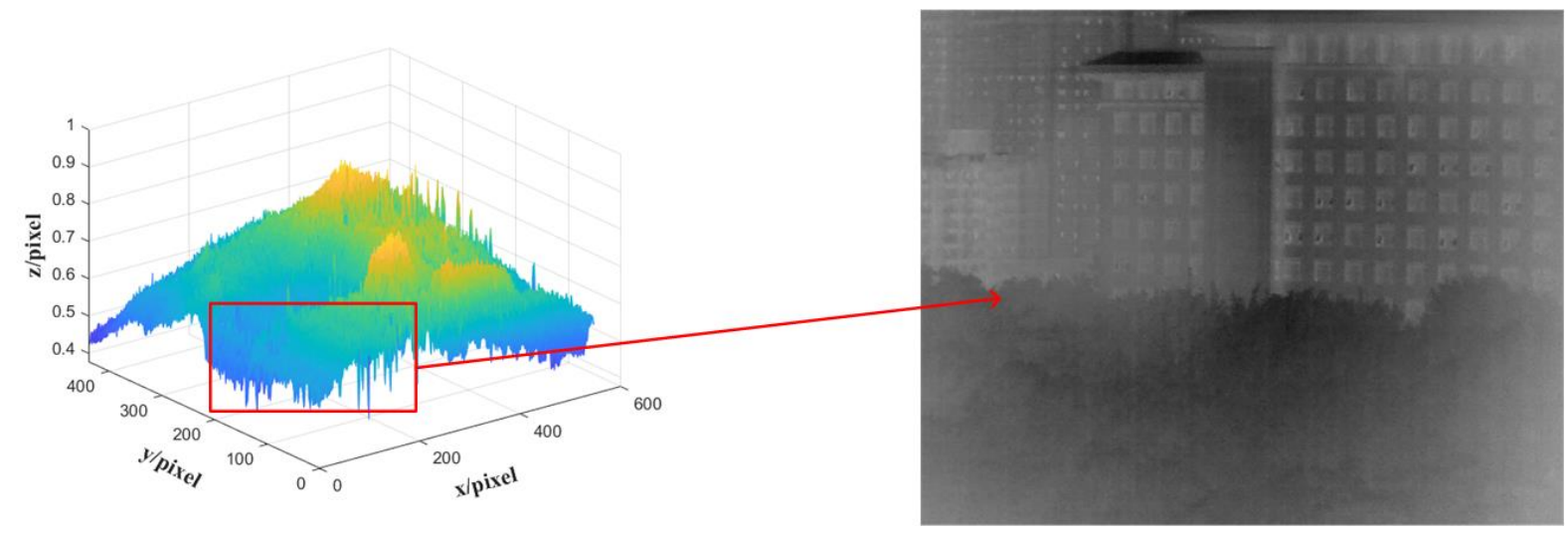

Figure 6. Comparison of removal effect of infrared-polarization cold reflection

(a) Original infrared-polarization image (b) Corrected infrared-polarization image

In order to show that DCT preprocessing can improve the performance of image, equations (7) and fireworks algorithm are used to calculate the DOLP image and fusion image. Compared with figure.7(a) and figure.7(b), it can be found that the subjective visual effect of DCT preprocessed image results is significantly better than the original results. Among them, the shape of the house in the distance is highlighted in the light-intensity image, the edge contour of the DOLP image is more obvious, the boundary of ceramic tile in the fusion image is much clearer, and the noise is less.

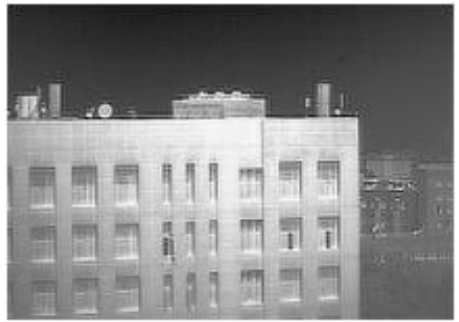

$\mathrm{S}_{0}$

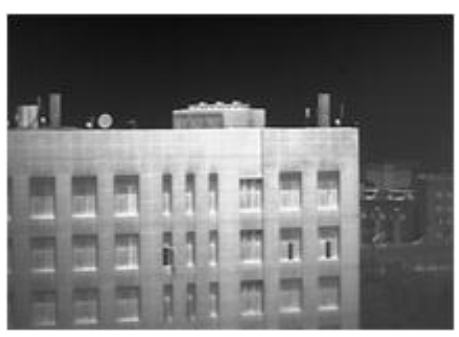

$\mathrm{S}_{0}$

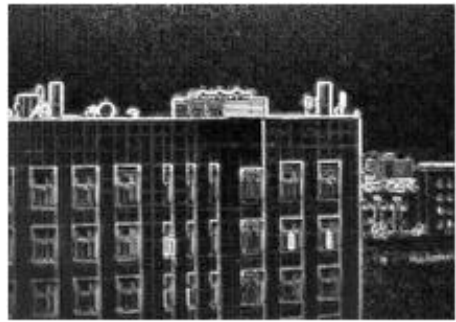

DOLP

(a)

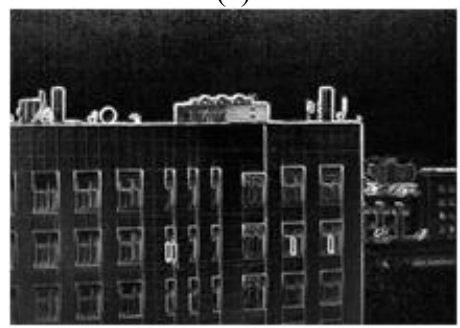

DOLP

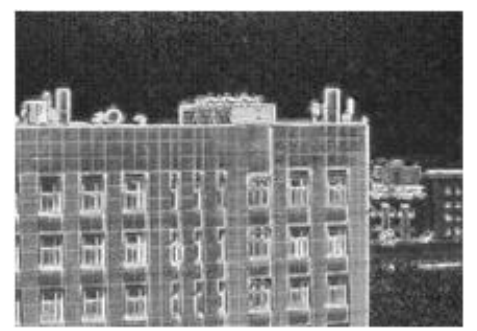

FWA

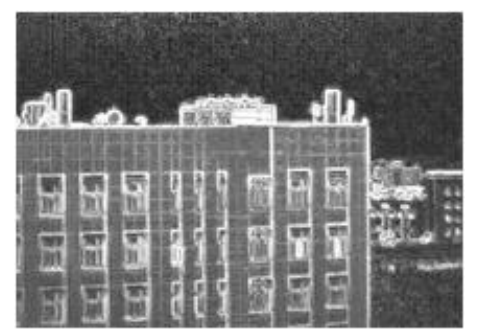

FWA

(b)

Figure 7. Polarization characteristic image and FWA fusion image. 
(a) The original images;(b) DCT preprocessed images;

As can be seen from Table 1, the performance of the preprocessed images has been improved to different extents, and the indexes of AG, STD and SF have been improved greatly, which is in accordance with the law of high-frequency and low-frequency processing performance of the images, the results show that there is not too much other information, which has little effect on the information of the original image. Therefore, the DCT preprocessing method can reduce the sensitivity of the image, reduce the noise and improve the robustness of the images.

Table 1. Image performance comparison

\begin{tabular}{|c|c|c|c|c|c|c|c|c|c|c|c|c|}
\hline \multirow{2}{*}{ Value } & \multicolumn{3}{|c|}{ AG } & \multicolumn{3}{|c|}{ STD } & \multicolumn{3}{|c|}{ SF } & \multicolumn{3}{|c|}{ IE } \\
\hline & I & DOLP & FWA & I & DOLP & FWA & I & DOLP & FWA & I & DOLP & FWA \\
\hline $\mathrm{a}$ & 6.5452 & 13.0714 & 13.7463 & 64.8397 & 46.0214 & 47.1012 & 22.6984 & 41.7238 & 36.5739 & 7.6816 & 6.4740 & 7.2756 \\
\hline $\mathrm{b}$ & 7.3803 & 15.7042 & 15.8911 & 66.9076 & 52.7612 & 56.9991 & 24.5208 & 47.0225 & 39.0387 & 7.7492 & 6.8549 & 7.5858 \\
\hline Rate & $12.6 \%$ & $20.1 \%$ & $15.6 \%$ & $3.1 \%$ & $12.8 \%$ & $21.0 \%$ & $8.3 \%$ & $12.7 \%$ & $7.7 \%$ & $0.9 \%$ & $5.9 \%$ & $4.3 \%$ \\
\hline
\end{tabular}

\subsection{Fusion experiments and quality analysis}

To validate verification infrared-polarization image fusion algorithm proposed in this paper, the experiment selects three groups of the infrared image and the DOLP image intensity under the natural background, which is later compared with several other methods, such as Brovery transform fusion algorithm (Brovery), principal component transformation fusion algorithm (PCA), Laplace pyramid fusion algorithm (Laplace), discrete wavelet transformation fusion algorithm (DWT), variance based fusion algorithm (Var). 

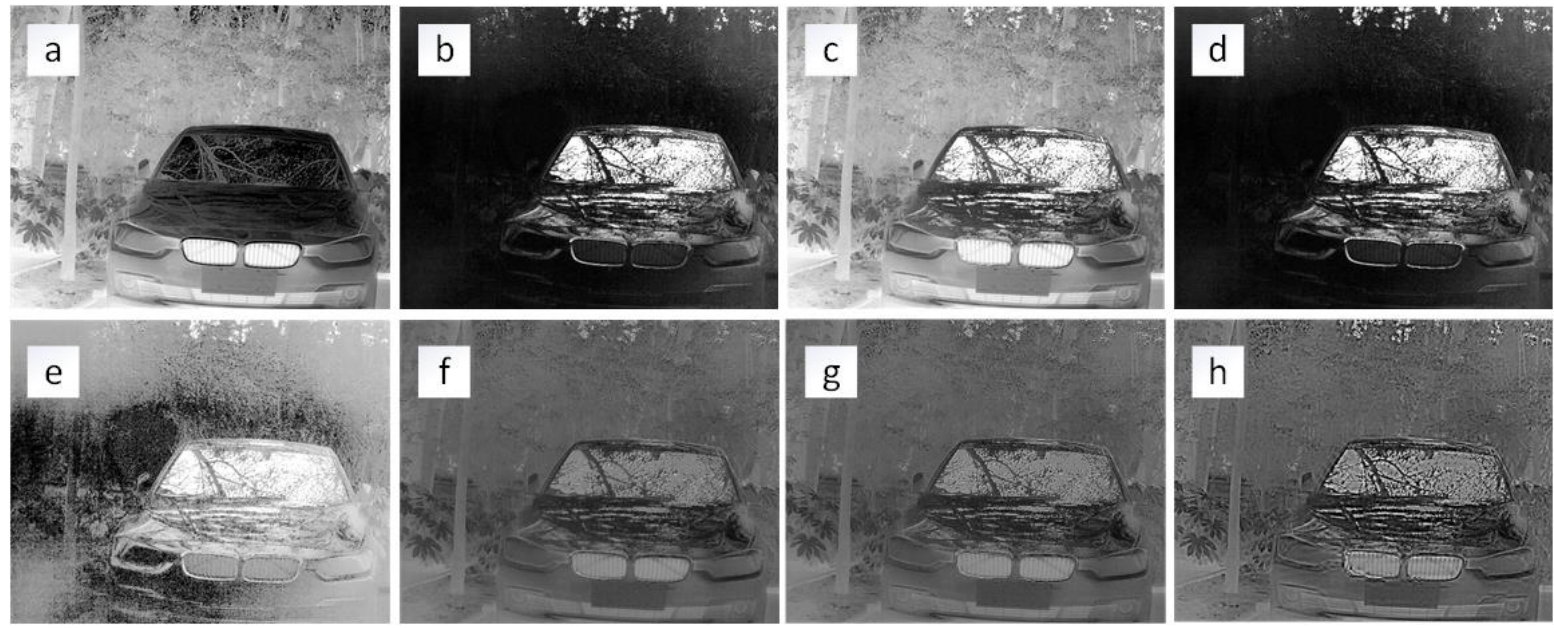

Figure 8. Fusion results of Car image

Figure 8 shows the infrared-intensity image and DOLP image of the car (Car) obtained by the long-wave infrared-polarization imaging system. The infrared-intensity image clearly shows the temperature difference of various parts of the car. The higher temperature of the car's intake grille can be easily seen. From the DOLP image, the reflection of the tree on the Car's glass and smooth front baffle can be distinguished, and the gap between the tree tops at the back of the car is also obvious. From the fusion results, FWA can better integrate the rich information of infrared-intensity image and DOLP image, and the important information on the air intake grille and glass is preserved. Although other algorithms can successfully fuse the image, the imaging visual effect is not good. By observing, it can be found that the images of Brovey and DOLP are similar, but more infrared information is lost; the fusion images of Laplace, DWT and Var are dark as a whole, which integrate the information of light intensity and DOLP, but the visual effect is poor; PCA fusion images produce more noise, and the fusion quality is the worst. 

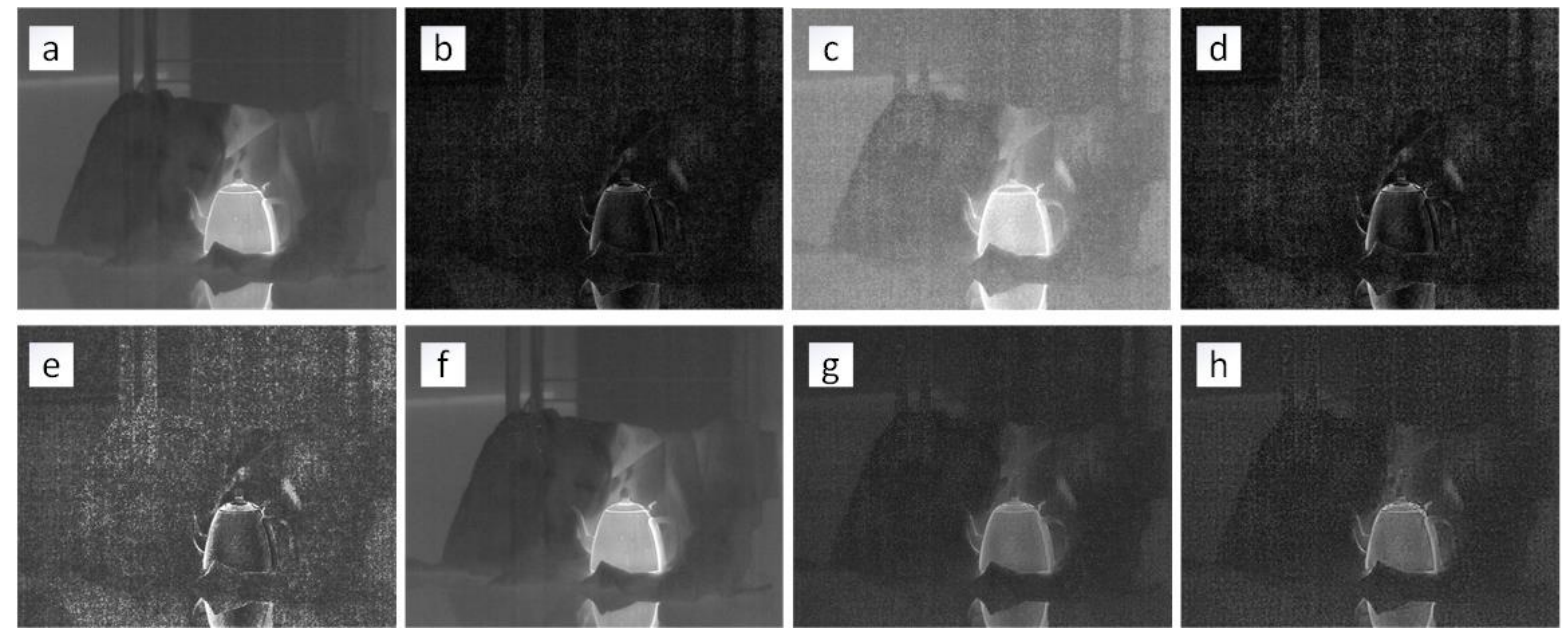

Figure 9. Fusion result of Kettle image

(a) S0; (b) DOLP; (c) FWA; (d) Brovery; (e) PCA; (f) Laplace;(g) DWT; (h) Var

Figure 9 shows the fusion result of infrared-intensity image and DOLP image of hot kettle (Kettle). In the infrared-intensity image, the infrared information of Kettle is stronger, while in the infrared-polarization image, the handle and the plastic part of Kettle have better contrast. From the perspective of subjective visual effect, all the six algorithms can fuse the main feature information of the source image, FWA and Laplace fusion images have better clarity, but Laplace fusion image loses most of the DOLP information; Brovey and PCA fusion images lose more infrared information, the contrast and overall gray value are low; DWT and Var fusion images produce unsatisfactory gray area, poor visual effect.
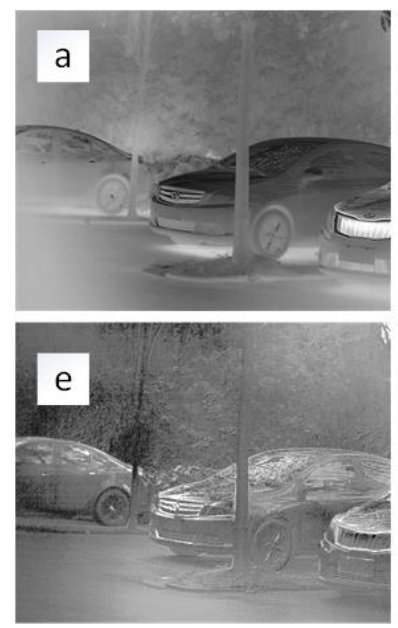
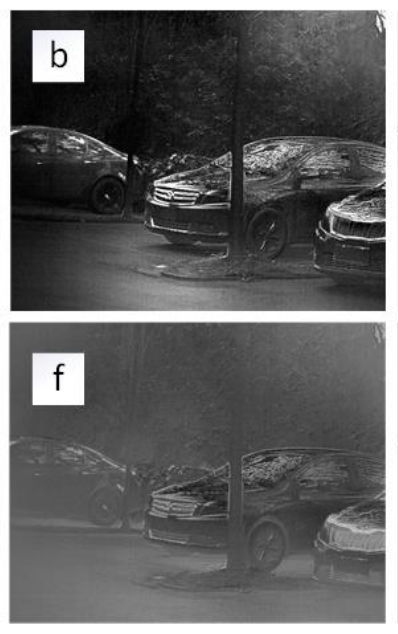
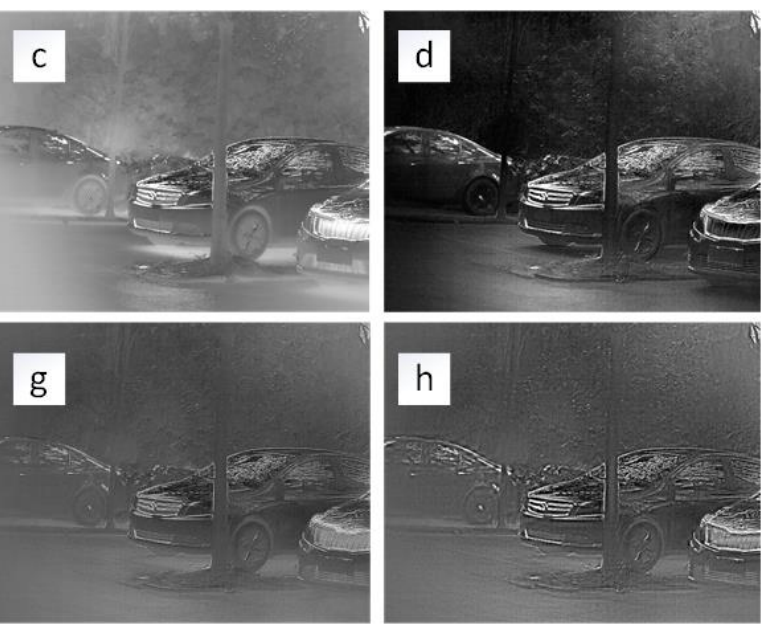

Figure 10. Fusion results of Three Cars image 
(a) S0; (b) DOLP; (c) FWA; (d) Brovery; (e) PCA; (f) Laplace;(g) DWT; (h) Var

Figure 10 shows the fusion result of infrared-intensity image and polarization degree image of three outdoor cars (Three Cars). The infrared-intensity image depicts the overall appearance of Three Cars, while the edge contour of Three Cars is highlighted in the DOLP image, and the frame and glass are distinguished from each other. Compare to other fusion methods, FWA has the best visual effect, which can better fuse the information of infrared-intensity image and DOLP image, for example, the residual temperature at the bottom of the middle vehicle is well preserved; Brovery fusion image loses more infrared information; PCA and Var fusion image mainly retains the contour information of three vehicles, but most areas are fuzzy; Laplace and DWT fusion image quality is poor Good, dark as a whole, not distinguishable from the background.

Table 2. Objective evaluation of the fusion results of Car

\begin{tabular}{lllllll}
\hline & STD & SF & MI & SSIM & PSNR & IE \\
\hline FWA & 59.0338 & $\mathbf{3 8 . 8 8 3 5}$ & 0.7725 & $\mathbf{1 . 0 2 2 0}$ & $\mathbf{3 5 . 8 5 8 9}$ & 6.9998 \\
\hline Brovery & 23.9146 & 19.8501 & 0.2835 & 0.6358 & 26.6682 & 6.5403 \\
\hline PCA & $\mathbf{6 1 . 2 2 8 9}$ & 31.7733 & 0.7723 & 0.4034 & 15.9042 & 6.3150 \\
\hline Laplace & 49.3024 & 36.0684 & $\mathbf{0 . 8 6 1 9}$ & 1.0108 & 18.9425 & $\mathbf{7 . 4 1 9 2}$ \\
\hline DWT & 28.7084 & 34.8721 & 0.6887 & 0.9275 & 22.3346 & 6.7598 \\
\hline Var & 34.5131 & 37.8141 & 0.7078 & 0.8925 & 22.0146 & 6.9650 \\
\hline
\end{tabular}

Table 3. Objective evaluation of the fusion results of Kettle

\begin{tabular}{lllllll}
\hline & STD & SF & MI & SSIM & PSNR & IE \\
\hline FWA & 26.8064 & 25.5317 & $\mathbf{1 . 9 7 0 3}$ & $\mathbf{1 . 6 8 8 2}$ & $\mathbf{4 1 . 3 6 9 1}$ & $\mathbf{6 . 2 7 9 4}$ \\
\hline Brovery & 20.4560 & $\mathbf{2 6 . 5 3 7 7}$ & 1.2698 & 1.0659 & 39.5354 & 5.7909 \\
\hline PCA & $\mathbf{2 7 . 8 0 0 4}$ & 25.6799 & 1.1005 & 0.9885 & 29.9453 & 5.5864 \\
\hline Laplace & 26.7505 & 15.4518 & 1.4195 & 1.1806 & 38.4376 & 6.0711 \\
\hline DWT & 17.6978 & 17.9242 & 0.8892 & 1.1236 & 36.0129 & 5.9309 \\
\hline Var & 21.2395 & 23.3096 & 1.4948 & 0.9349 & 34.4954 & 5.0816 \\
\hline
\end{tabular}

Table 4. Objective evaluation of the fusion results of Three Cars

\begin{tabular}{lllllll}
\hline & STD & SF & MI & SSIM & PSNR & IE \\
\hline FWA & $\mathbf{4 3 . 4 0 8 1}$ & $\mathbf{2 9 . 1 1 1 9}$ & 0.9252 & 1.1722 & $\mathbf{2 9 . 5 5 2 2}$ & $\mathbf{7 . 0 5 9 5}$ \\
\hline Brovery & 20.8235 & 14.0456 & $\mathbf{0 . 9 9 1 0}$ & $\mathbf{1 . 2 3 5 2}$ & 26.2675 & 6.2853 \\
\hline PCA & 34.2784 & 22.4001 & 0.8873 & 0.9243 & 24.3576 & 6.5864 \\
\hline Laplace & 32.2026 & 22.6499 & 0.7764 & 1.1011 & 28.2665 & 6.8635 \\
\hline DWT & 23.9684 & 25.1467 & 0.8480 & 1.1664 & 29.3347 & 6.4657 \\
\hline Var & 28.2766 & 28.4663 & 0.8379 & 1.1111 & 29.0003 & 6.6488 \\
\hline
\end{tabular}

As can be seen from the tables above Table 2, 3, 4 and Figure 11, the fireworks algorithm 
is slightly worse than the comparison methods in some fusion evaluation indexes, but on the whole it shows better superiority. The PSNR, SF, SSIM and IE are the best in 3 groups, and the stabilities of STD and MI are generally good. In Car image fusion experiment, our method is the best in SF, SSIM and PSNR, the fusion result of Laplace is slightly better than that of MI and IE, but the fusion image of Laplace has artifacts; the STD Index of this method is lower than that of PCA, but the PCA fusion image has more noise and distortion, and the proposed method is better than the comparison methods in general. In the experiment of Kettle image fusion, our method is the best in MI, SSIM, PSNR and IE, and PCA and Brovery are slightly better than this method in STD and SF respectively, but these two methods fluctuate greatly in other indexes, the overall performance is poor. In the experiment of Three Cars image fusion, Brovery method is slightly better than FWA in MI and SSIM indexes, but the performance of this method is not consistent, for example, the worst result is obtained in STD, SF and IE Indexes. Our method is the best in other indicators, and obtain the best visual effect.

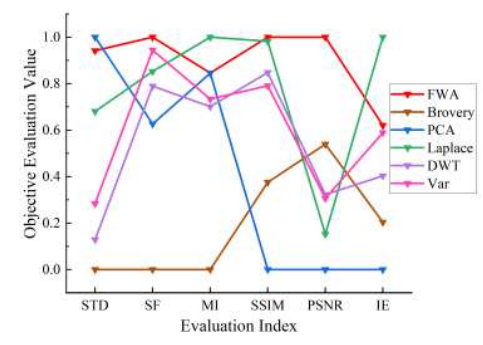

a

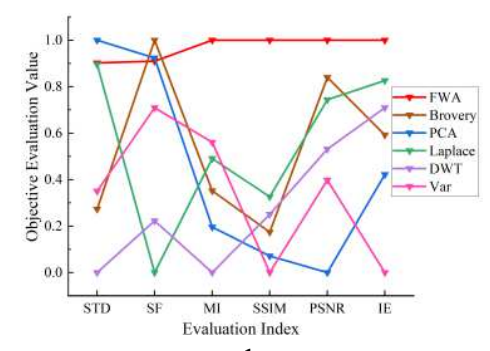

b

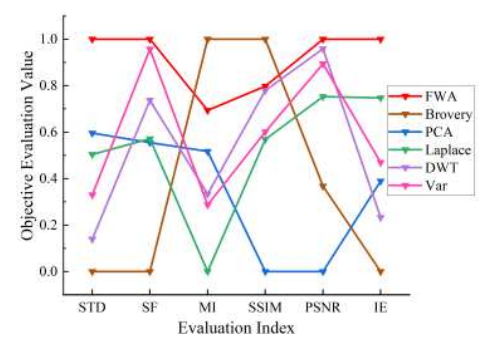

Figure 11. Contrast effect image of fusion image evaluation index homogenization.

(a) Car; (b) Kettle; (c) Three Cars

\subsection{Further analysis}

In this paper, the fitness function is constructed based on the root mean square error of the source image and fusion image, and the minimum root mean square error of the point is obtained when the number of iterations is satisfied. It can be seen from equation (22) that 
RMSE and PSNR are inversely proportional. The smaller RMSE is, the larger PSNR is, and the better the quality of the fusion image is. From the three groups of image fusion experiments, it can be seen that the PSNR index of the fusion image obtained by fireworks algorithm is better than that of those comparison methods, which verifies the feasibility of this method. The experimental results of infrared-polarization image fusion in different scenes show that this method is suitable for infrared- intensity and DOLP image fusion in complex environment, and its subjective visual effect and objective evaluation results are greatly improved.

\section{Conclusion}

Focusing on the characteristics of information redundancy and complementary features between infrared-intensity image and DOLP image, we propose a fusion method of infrared-polarization image based on fireworks algorithm. In order to improve the imaging quality of the source image, cold reflection and two-dimensional discrete cosine transform are used to process the polarization image. After preprocessing, the image performance is improved to various degrees. In the aspect of infrared-intensity image and DOLP image fusion, the minimum root mean square error is taken as the fitness function of fireworks algorithm, and the optimal weight of source image is calculated through the optimal fitness function to realize image fusion, and the subjective visual effect and objective index are evaluated. The experimental results show that the proposed method is superior to those comparison algorithms in subjective and objective evaluation indexes, by which the fusion results are good, the infrared characteristics and polarization characteristics of the source image are retained, and the imaging quality is greatly improved. 


\section{References}

[1] Wang B, Zhang B F \& Liu X W.et al. Novel infrared image enhancement optimization algorithm combined with DFOCS[J]. Optik224: 165476.

https://doi.org/10.1016/j.ijleo.2020.165476(2020)

[2] Zhang J, Shao J \& Chen J.et al. PFNet: an unsupervised deep network for polarization image fusion[J]. Optics letters45(6): 1507-1510.

https://doi.org/10.1364/OL.384189(2020)

[3] Zhang L, Yang F \& Ji L. Multi-scale fusion algorithm based on structure similarity index constraint for infrared polarization and intensity images[J]. IEEE Access5: 24646-24655.https://doi.org/10.1109/ACCESS.2017.2764101(2017)

[4] KONG X Y, ZHAO Y Q \& PENG Q N.et al. Infrared Polarization Image Enhancement Algorithm Based on Mutual Structure Regularization Constraint[J]. Acta Photonica Sinica49(5): 510001-0510001.

https://doi.org/ 10.3788/gzxb20204905.0510001(2020)

[5] Kanwal H, Akhtar M, Assam M.et al. MSEF-ImgSeg: An Intelligent Algorithm for Multi Scale Exposure Fusion Using Image Segmentation and GGIF[J]. IEEE Access8: 166060-166070. https://doi.org/10.1109/ACCESS.2020.3022811(2020)

[6] DAI L Y, LIU G \& XIAO G.et al. Infrared and Visible Image Fusion Based on FRC Algorithm [J/OL]. Control and Decision. http://doi.org/10.13195/jkzyjc.2020.0669(2020)

[7] Sengupta S, Basak S \& Peters R A. Particle Swarm Optimization: A survey of historical and 
recent developments with hybridization perspectives[J]. Machine Learning and Knowledge Extraction1(1): 157-191.

https://doi.org/10.3390/make1010010(2019)

[8] Rajeswari C, Santhi M. Modified flower pollination algorithm for optimizing FOPID controller and its application with the programmable n-level inverter using fuzzy logic[J]. Soft Computing, 2020: 1-19.

https://doi.org/10.1007/s00500-020-05305-9(2020)

[9] Tan Y. Fireworks algorithm[J]. Heidelberg, Germany: Springer, 2015, 10: 978-3.

[10] Zheng S, Tan Y. A unified distance measure scheme for orientation coding in identification[C]//2013 IEEE Third international conference on information science and technology (ICIST). IEEE, 2013: 979-985.

https://doi.org/10.1109/ICIST.2013.6747701(2013)

[11] Misra P R, Si T. Image segmentation using clustering with fireworks algorithm[C]//2017 11th International Conference on Intelligent Systems and Control (ISCO). IEEE, 2017: 97-102.https://doi.org/10.1109/ISCO.2017.7855961

[12] Zheng S, Janecek A \& Tan Y. Enhanced fireworks algorithm[C]//2013 IEEE congress on evolutionary computation. IEEE, 2013: 2069-2077.

https://doi.org/10.1109/CEC.2013.6557813(2013)

[13] Lu Q, Gui W \& Su M. A fireworks algorithm for the system-level fault diagnosis based on MM* model[J]. IEEE Access7: 136975-136985. https://doi.org/10.1109/ACCESS.2019.2942336(2019) 
[14] Cui Y, Guo J S \& Li-Wei C, et al. Fireworks Algorithm-Based Dimensionality Reduction for Hyperspectral Imagery Classification[J]. Journal of South China University of Technology (Natural Science) 45(3): 20.

https://doi.org/10.3969/j.issn.1000-565X.2017.03.003(2017)

[15] Bejinariu S I, Luca R \& Costin H. Nature-inspired algorithms based multispectral image fusion[C]//2016 International Conference and Exposition on Electrical and Power Engineering (EPE). IEEE, 2016: 010-015.

https://doi.org/10.1109/ICEPE.2016.7781293(2016)

[16] Semrau D, Killey R I \& Bayvel P. The Gaussian noise model in the presence of inter-channel stimulated Raman scattering[J]. Journal of Lightwave Technology36(14): 3046-3055. https://doi.org/10.1364/JLT.36.003046(2018)

\section{Acknowledgement}

This study was supported by the National Natural Sciences Foundation of China (grant no.61505254)

\section{Author contributions}

\section{Competing interests}

The authors declare no competing interests. 
Figures

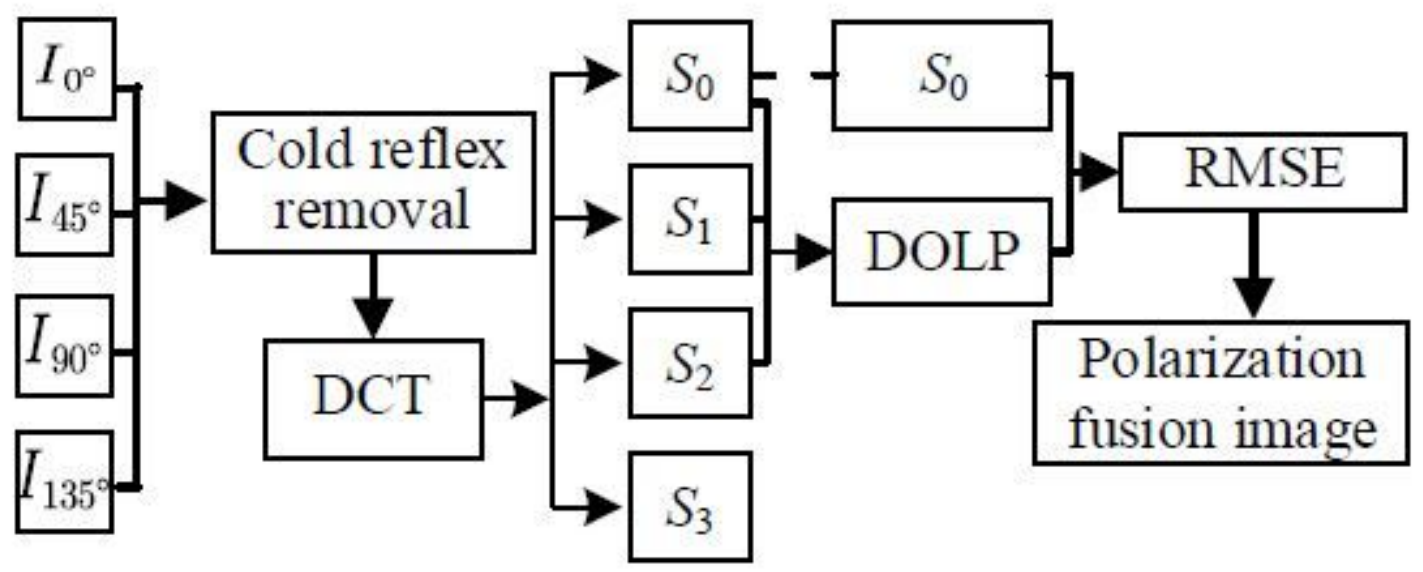

Figure 1

Flow chart of infrared-polarization image fusion based on firework algorithm

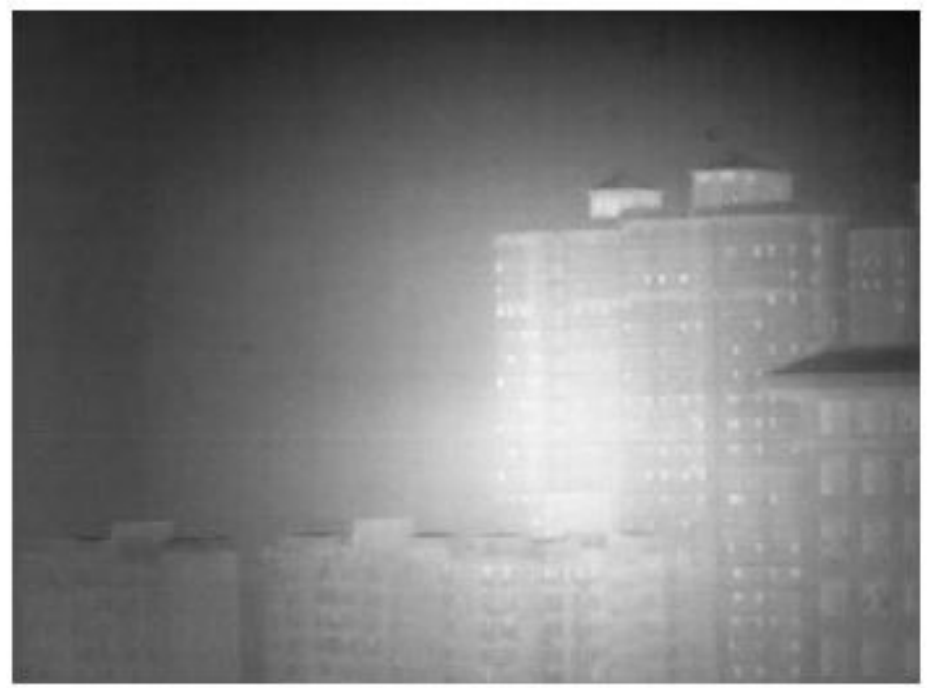

Figure 2

Cold reflection phenomenon of long-wave infrared-polarization imaging

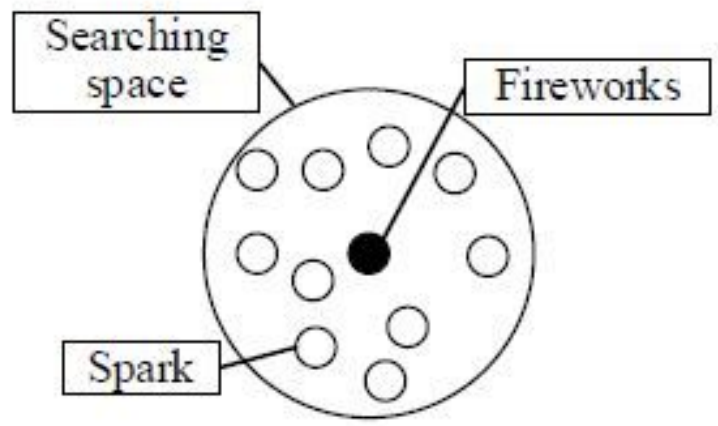


Figure 3

Sparks from fireworks explosions

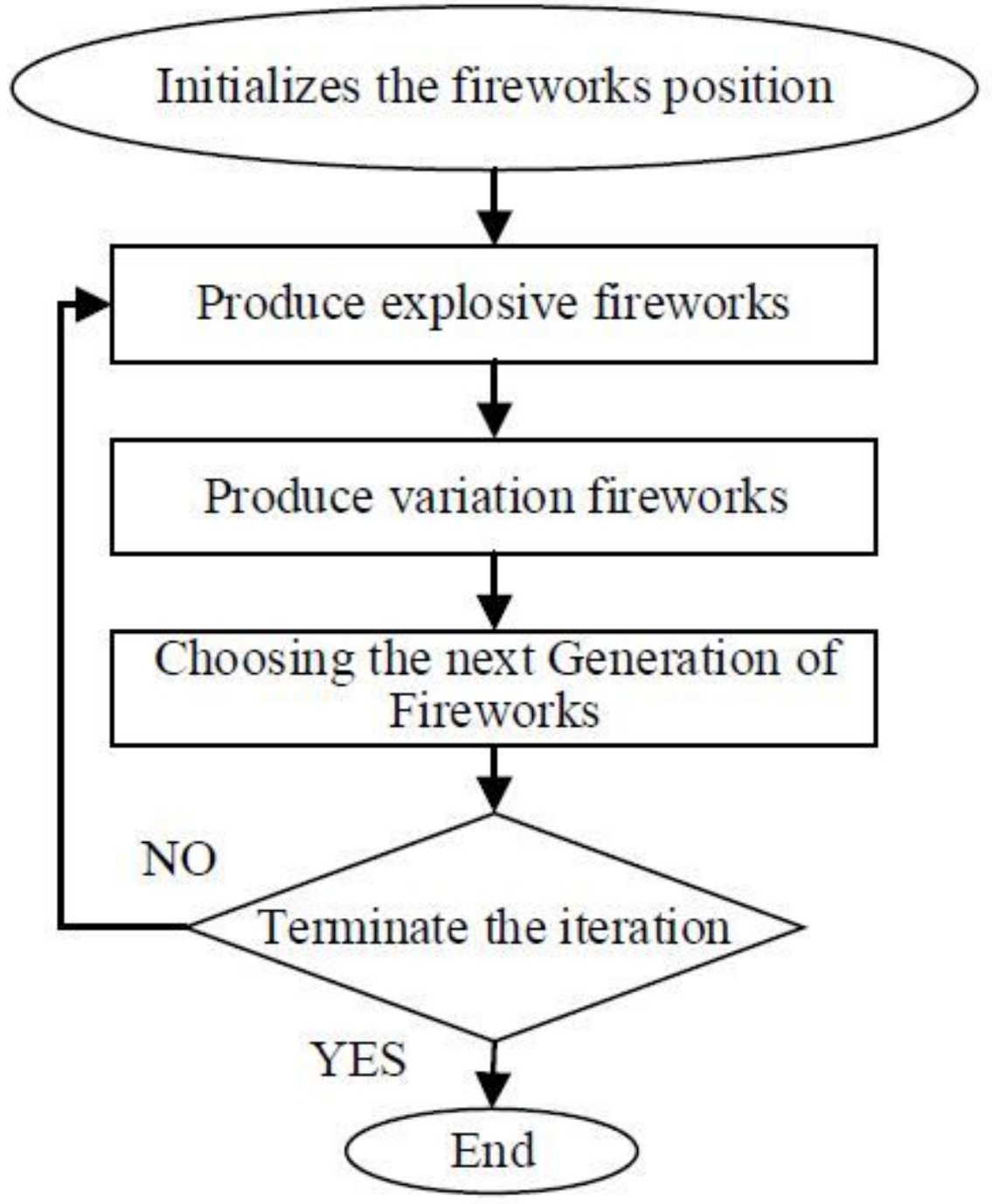

Figure 4

Flow chart of optimization algorithm 


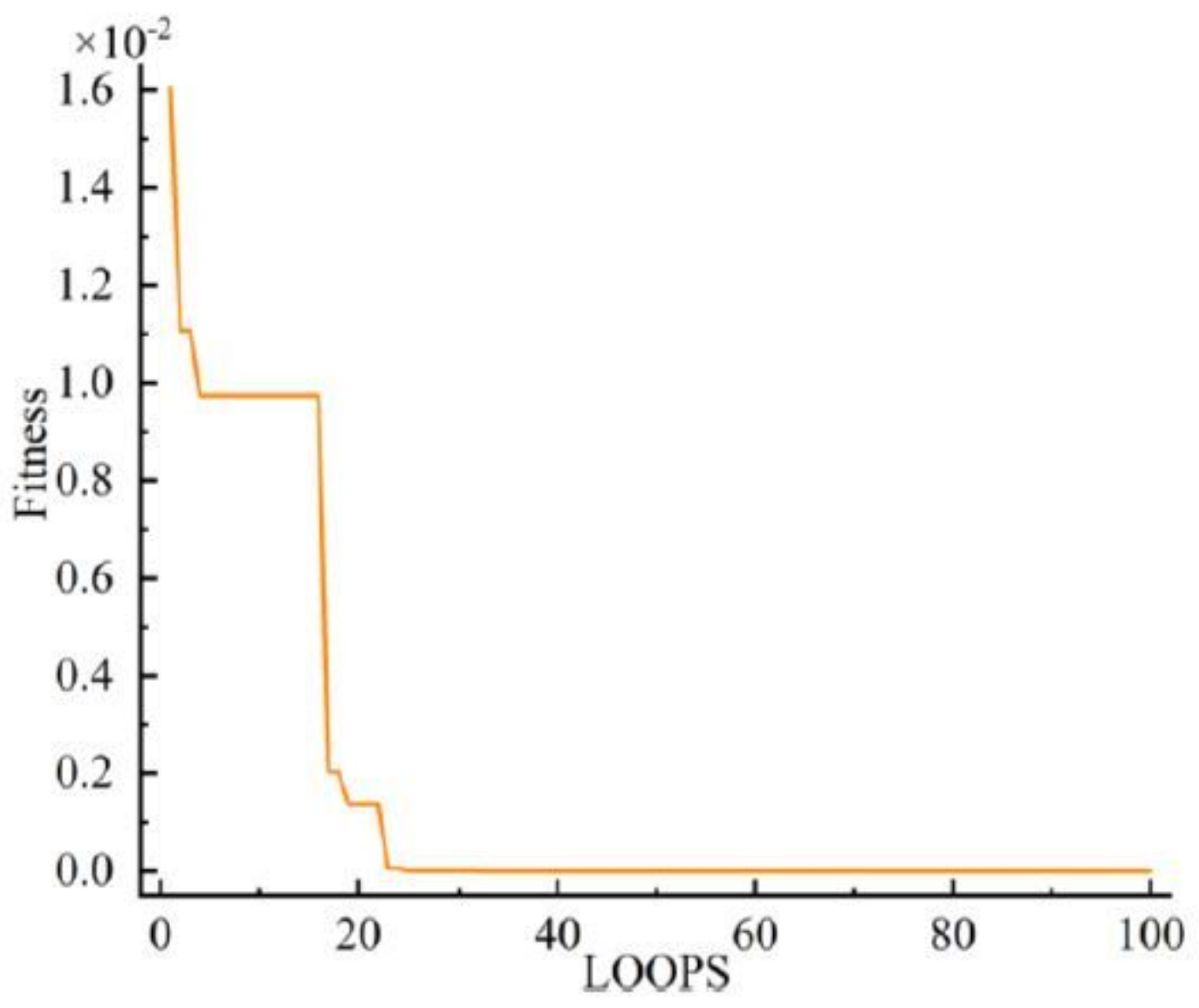

Figure 5

Optimization process of RMSE fitness 

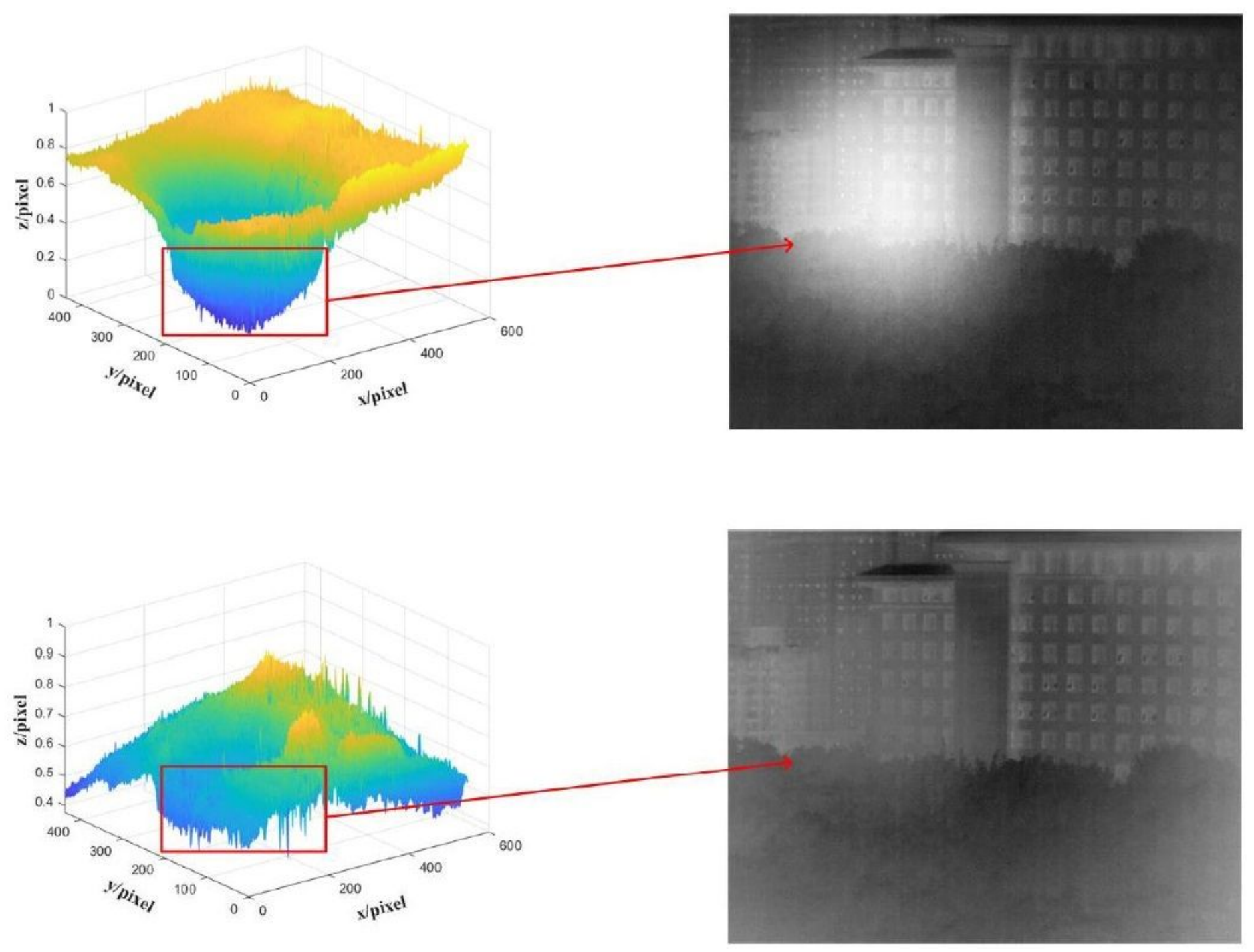

Figure 6

Comparison of removal effect of infrared polarization cold reflection (a) Original infrared polarization image (b) Corrected infrared polarization image 


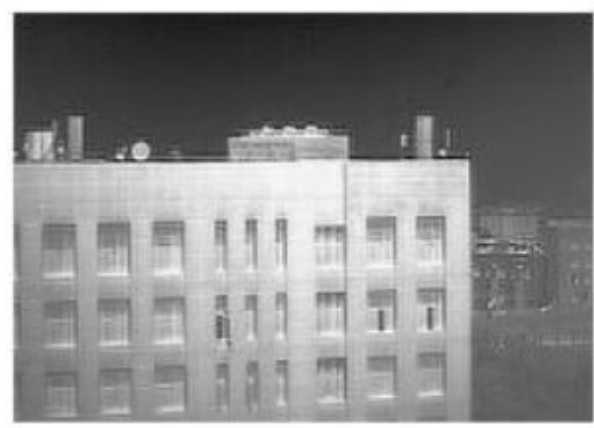

$\mathrm{S}_{0}$

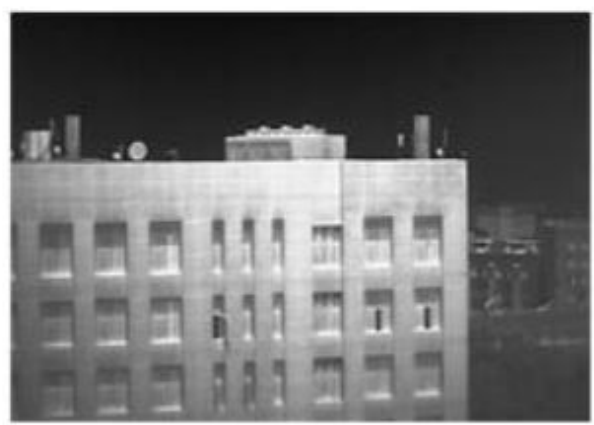

$\mathrm{S}_{0}$

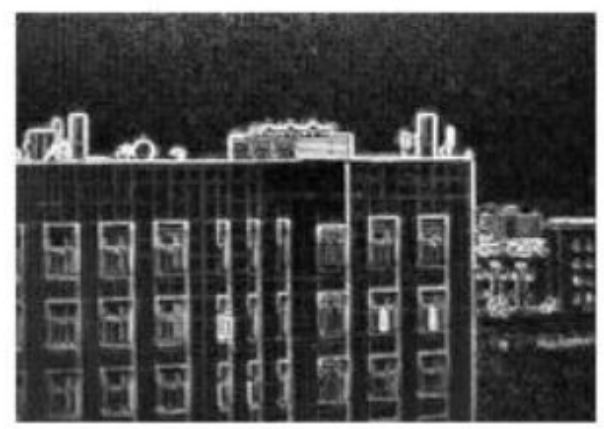

DOLP

(a)

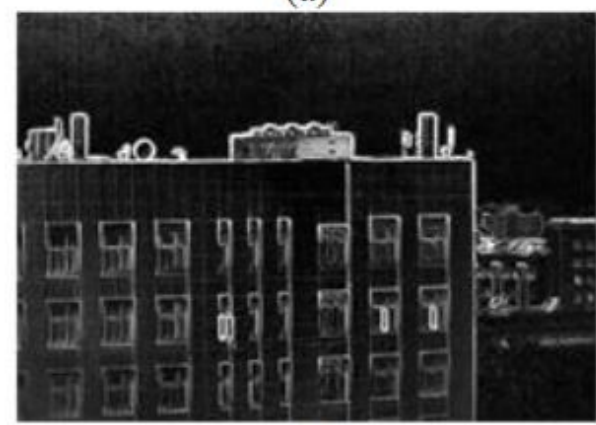

DOLP

(b)

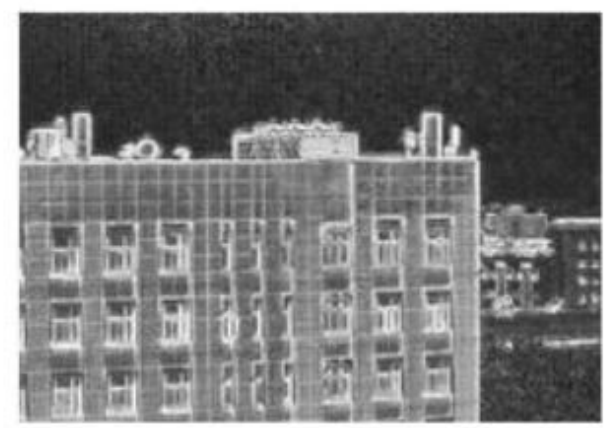

FWA

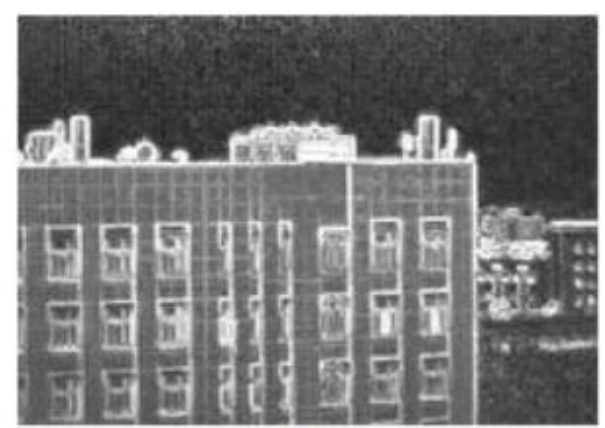

FWA

\section{Figure 7}

Polarization characteristic image and FWA fusion image. (a) The original images;(b) DCT preprocessed images;
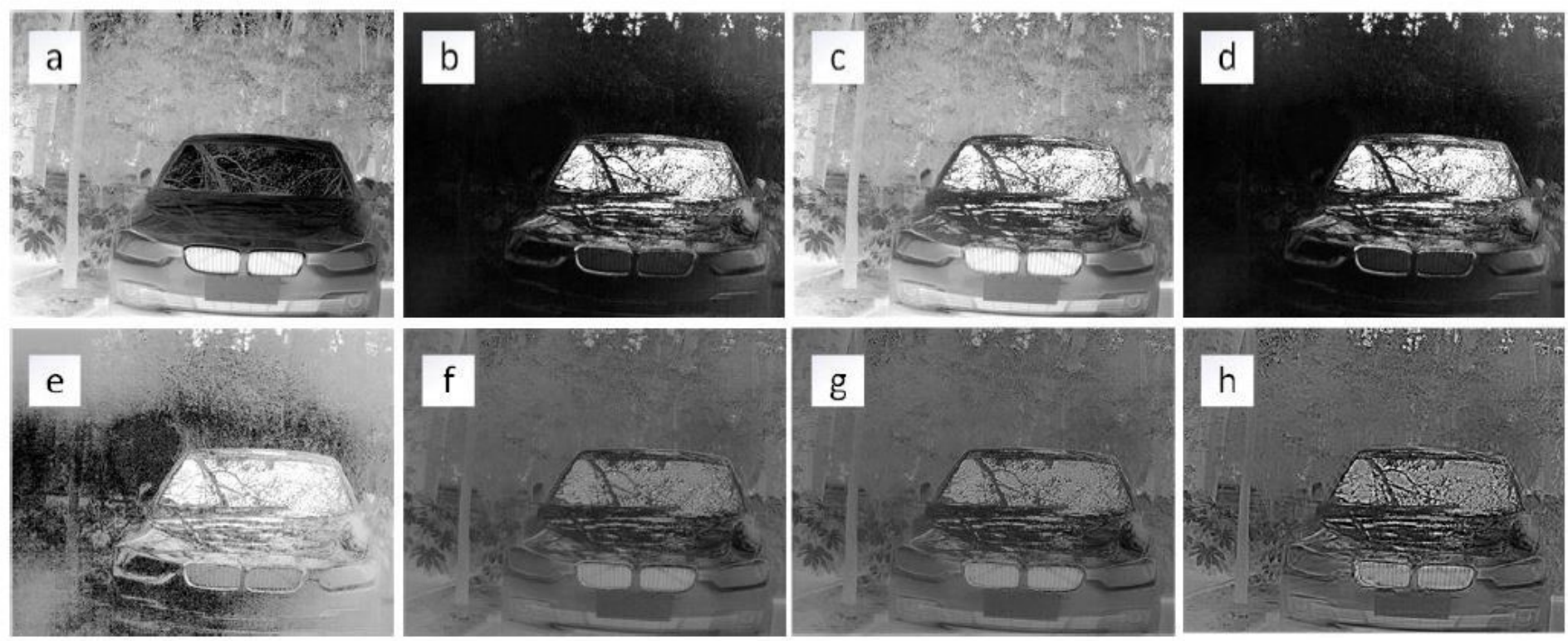

\section{Figure 8}

Fusion results of Car image (a) S0; (b) DOLP; (c) FWA; (d) Brovery; (e) PCA; (f) Laplace;(g) DWT; (h) Var 

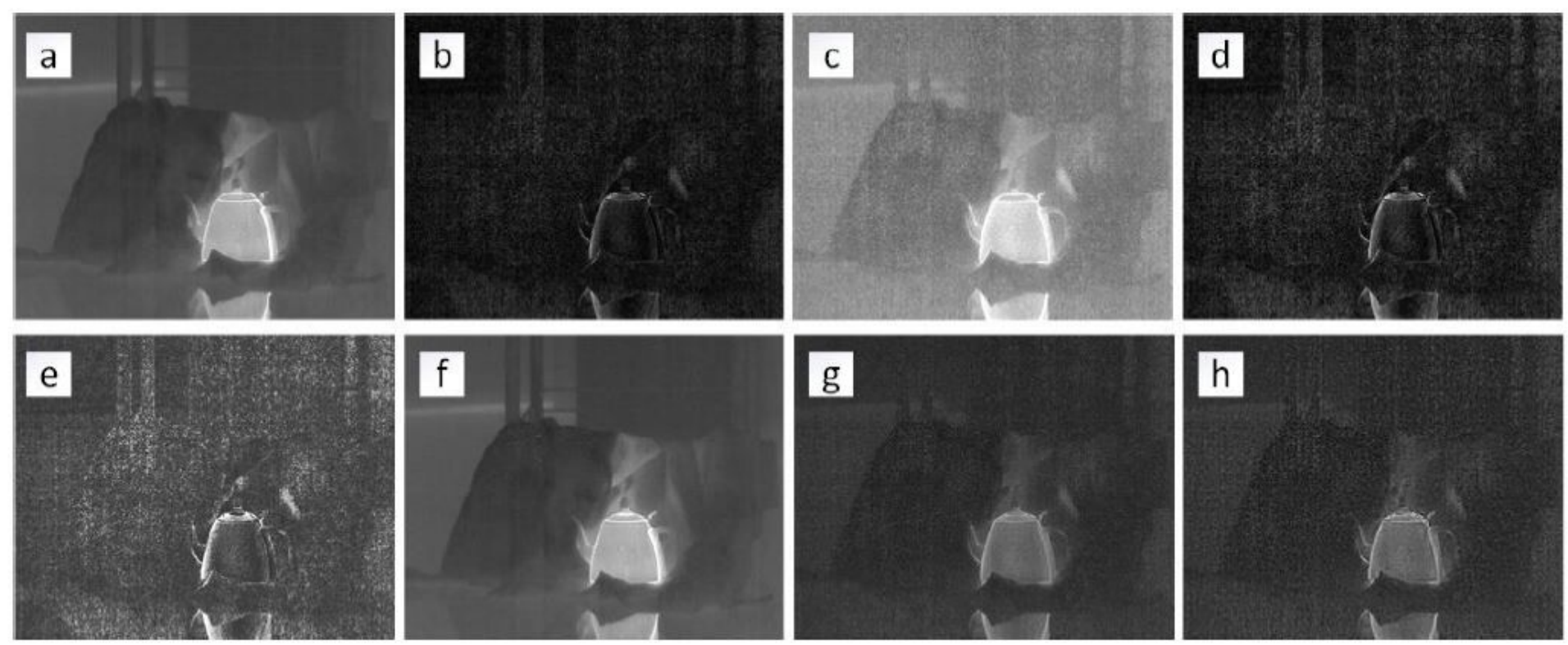

\section{Figure 9}

Fusion result of Kettle image (a) SO; (b) DOLP; (c) FWA; (d) Brovery; (e) PCA; (f) Laplace;(g) DWT; (h) Var
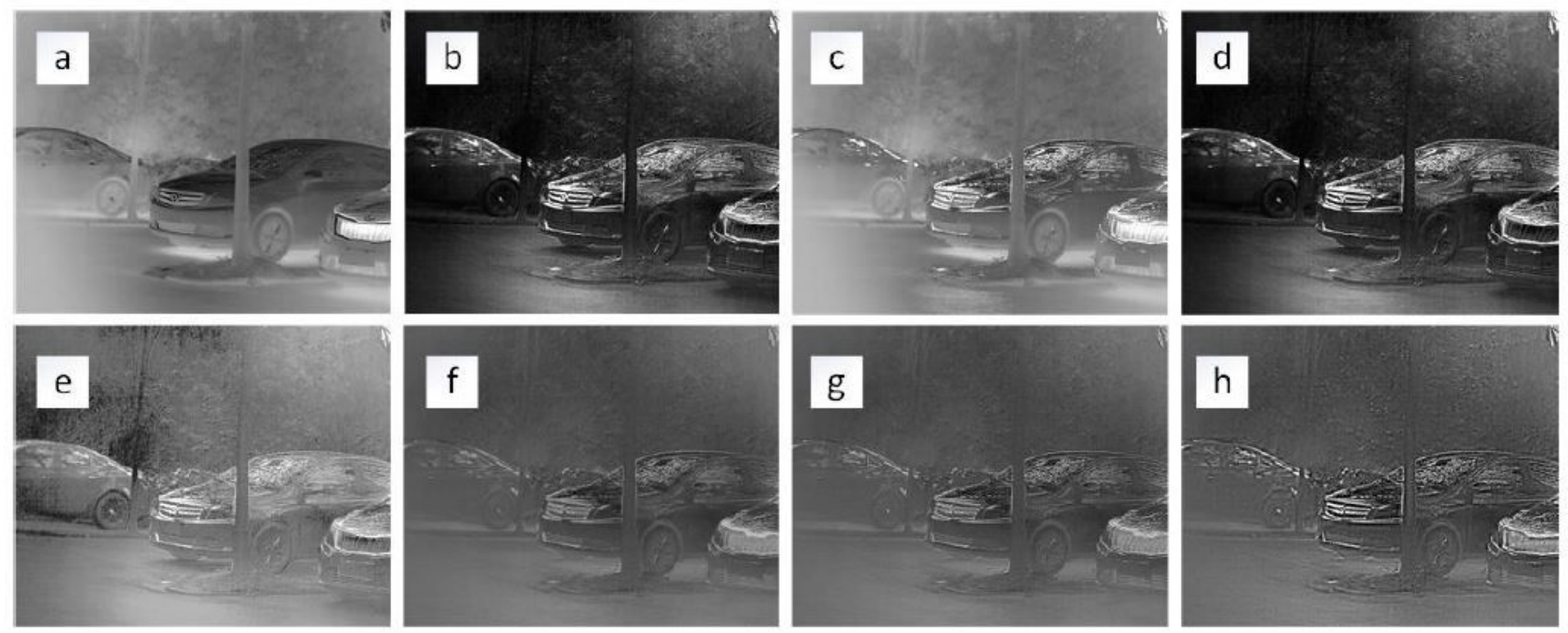

\section{Figure 10}

Fusion results of Three Cars image (a) S0; (b) DOLP; (c) FWA; (d) Brovery; (e) PCA; (f) Laplace;(g) DWT; (h) Var 


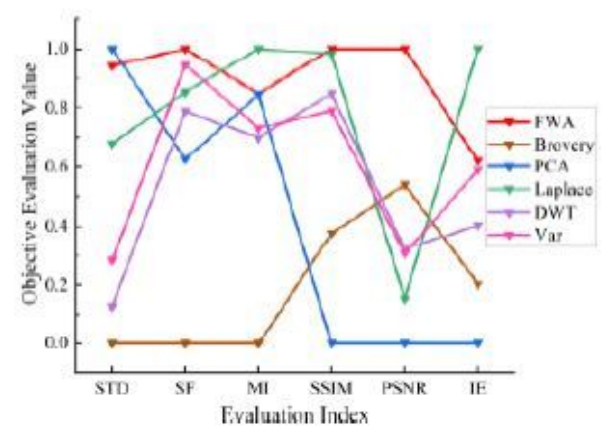

a

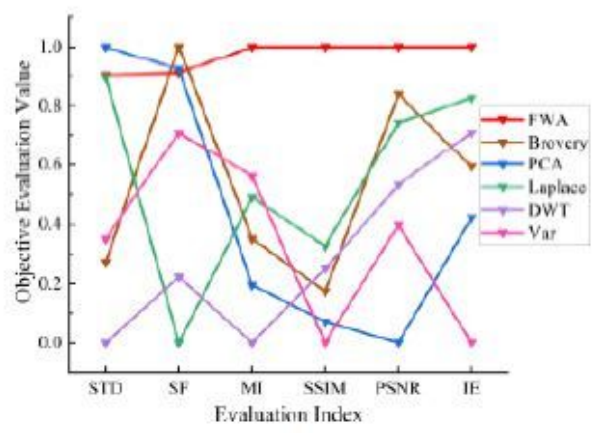

b

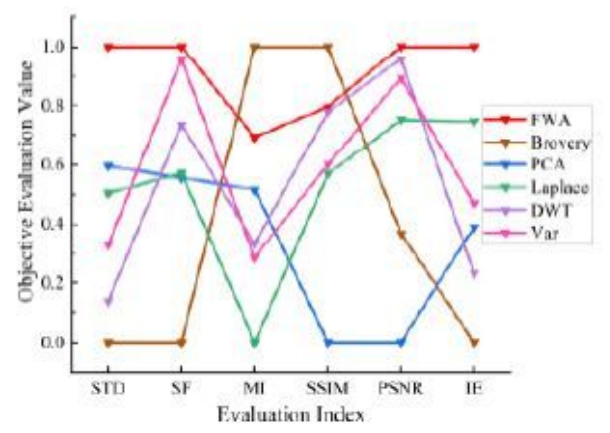

c

Figure 11

Contrast effect image of fusion image evaluation index homogenization. (a) Car; (b) Kettle; (c) Three Cars 